The response of human muscle triceps brachii (TB) to training, which consisted of skiing with sledges 500 miles over a period of 36 days, has been investigated by means of histochemical fiber typing (myofibrillar ATPase, $n=7$ ). Muscle biopsies were taken in the right TB during pretraining, and in the right as well as in the left TB after training. The percentage of type 2 fibers and intermediate fibers (type $2 \mathrm{C}$ and $1 \mathrm{~B}$ ) in the right TB decreased and increased, respectively, by $13 \%$. The fiber type distribution in the left TB after training was very similar to that in the right TB after training. This concordance indicates that the change in fiber type distribution was a result of the training per se, and not related to any eventual effect of the biopsy-sampling.

MUSCLE \& NERVE

6:553-556

1983

\title{
INCREASES IN MYOFIBRILLAR ATPaSe INTERMEDIATE HUMAN SKELETAL MUSCLE FIBERS IN RESPONSE TO ENDURANCE TRAINING
}

\author{
PETER SChANTZ, MPE, and JAN heNRIKSSON, MD, PhD
}

In a recent report in this journal, ${ }^{24}$ the authors described an increased percentage of myofibrillar ATPase intermediate fibers (type $2 \mathrm{C}$ and $1 \mathrm{~B}$ ) in the human triceps brachii muscle (TB) in response to prolonged endurance training. Based on these results and on information about the nature of myofibrillar A'TPase intermediate fibers, ${ }^{2,14-16,20,22}$ the increased fraction of intermediate fibers was interpreted as a sign of ongoing fiber type transformation.

The authors could not, however, entirely rule out that the increase of intermediate fibers was due to repeated biopsy-sampling which caused nerve damage, followed by reinnervation, ${ }^{15.19}$ or fiber damage. ${ }^{13,23}$ Although this seemed unlikely, since there were no signs of muscle denervation (atrophy), reinnervation (type grouping), or muscle fiber regeneration (myotubes), ${ }^{12,18,23}$ it was considered necessary to examine this possibility. A control study was, therefore, undertaken. The fiber

From the Department of Physiology III, Karolinska Institutet, Stockholm Sweden.

Acknowledgments: We wish to express our gratitude to the members of the Minnesota-Lappland Expedition, 1982, at Concordia College, MN for the opportunity to perform this study in connection with their tour, as well as to Ms. A.-B. Olrog, A. M. Gullström, and U. Siltberg for excellent technical and secretarial assistance. This study was supported by grants from the Karolinska Institute's Research Funds.

Address reprint requests to Dr. P. Schantz at the Department of Physiology III, Karolinska Institutet, Lindingövägen 1, S-114 33 Stockholm, Sweden.

Received for publication December 20, 1982; revised manuscript accepted for publication May 6, 1983

0148-639x/0608/0553 $\$ 01.25 / 0$

- 1983 John Wiley \& Sons, Inc type distribution was studied in the right TB before prolonged endurance training, and in the right and left $\mathrm{TB}$ after training.

\section{MATERIALS AND METHODS}

subjects. Four healthy, moderately trained women and three men participated in the study. Average age, height, and weight for the women was 25 years (range $20-29), 1.62 \mathrm{~m}(1.60-1.70)$, and 58 $\mathrm{kg}(50-64)$, respectively, and for the men 27 years (24-29), $1.81 \mathrm{~m}(1.73-1.98)$, and $80 \mathrm{~kg}(68-98)$, respectively. They were informed about the procedure and the risks involved in the experiments before they volunteered to participate. The study was approved by the Committee of Ethics at the Karolinska Institutet.

Tralning. The training consisted of approximately 500 miles $(800 \mathrm{~km})$ of skiing in mountain terrain. Teams of two skiers pulled a load $(80 \mathrm{~kg})$ on one sledge. The distance was covered in 36 days of skiing with 5 days of rest evenly interspersed.

Musclo sampllng and stalning. Muscle biopsies were obtained from the middle-lower portion of the medial head of TB using the needle biopsy technique. ${ }^{1}$ Biopsies were taken from the right TB before and after training, as well as from the left TB after training. The posttraining biopsies were taken 5 days after termination of the training. Serial transverse sections of the muscle samples were stained for myofibrillar ATPase activity. ${ }^{10,21}$ The fibers were classified into fiber types 1 and $2^{8}$ and 
into the subgroups $2 \mathrm{~A}$ and $2 \mathrm{~B},{ }^{4}$ type $2 \mathrm{C},{ }^{5.6}$ and 1B. ${ }^{7}$ Type $2 \mathrm{C}$ and $1 \mathrm{~B}$ fibers will be referred to as intermediate fibers. On the average, 420 (range 130-920) fibers were counted from each muscle before and after training. The handling of the biopsies, the staining procedure, as well as the fiber type classification has been described in detail elsewhere. $^{24}$

Statistics. Results are presented as means and standard error of means or ranges. A two-way analysis of variance (ANOVA) of no difference in means was applied to the data on fiber type distribution. Tukey's method of multiple comparisons ${ }^{17}$ was applied when the ANOVA indicated an overall significance. The magnitude of the variance differed for the percentage of intermediate fibers before and after training, hence the ANOVA could not be applied on this portion of fibers. Instead, Wilcoxon's signed rank sum test was used for comparisons. In that test, because the different portions of intermediate fibers were used in two comparisons, a higher significance level $(P<0.025)$ was chosen. In other tests, a probability level of less than 0.05 was considered significant.

\section{RESULTS}

The muscle fiber type distribution before and after training is listed in Table 1. No change occurred in training with respect to the type 1 fibers, while the percentage of type 2 fibers $(2 A+2 B)$ decreased from $69 \%$ before training to $56 \%$ after training in the right TB. The same percentage $(56 \%)$ was seen in the left TB after training. It differed significantly from the percentage of type 2 fibers seen in the right TB at pretraining. The decrease in type 2 fibers was associated with an increase in intermediate fibers (IM) from $2 \%$ to $15 \%$. The percentage of IM fibers in the left TB after training was similar to that in the right TB (14\%), but did not differ significantly from the percentage of IM fibers seen in the right TB at pretraining. Individual changes in

Table 1. Muscle fiber type distribution (\%) in TB before and after training (means \pm SEM).

\begin{tabular}{lcrrr}
\multirow{2}{*}{$\begin{array}{l}\text { Fiber } \\
\text { types }\end{array}$} & \multicolumn{2}{c}{ Before training } & \multicolumn{2}{c}{ After training } \\
\cline { 2 - 3 } \cline { 4 - 5 } & \multicolumn{1}{c}{ right } & & \multicolumn{1}{c}{ right } & left \\
\hline 1 & $29 \pm 3$ & & $28 \pm 2$ & $30 \pm 3$ \\
Intermediate & $2 \pm 1$ & & $* 15 \pm 4$ & $14 \pm 4$ \\
$2 A$ & $48 \pm 4$ & & $42 \pm 5$ & $37 \pm 4$ \\
$2 B$ & $21 \pm 5$ & $14 \pm 3$ & $19 \pm 5$ \\
$(2 A+2 B)$ & $69 \pm 3$ & $* 56 \pm 5$ & $* 56 \pm 5$ \\
\hline
\end{tabular}

-Denotes significant differences $(P<0.05)$ versus pretraining values.

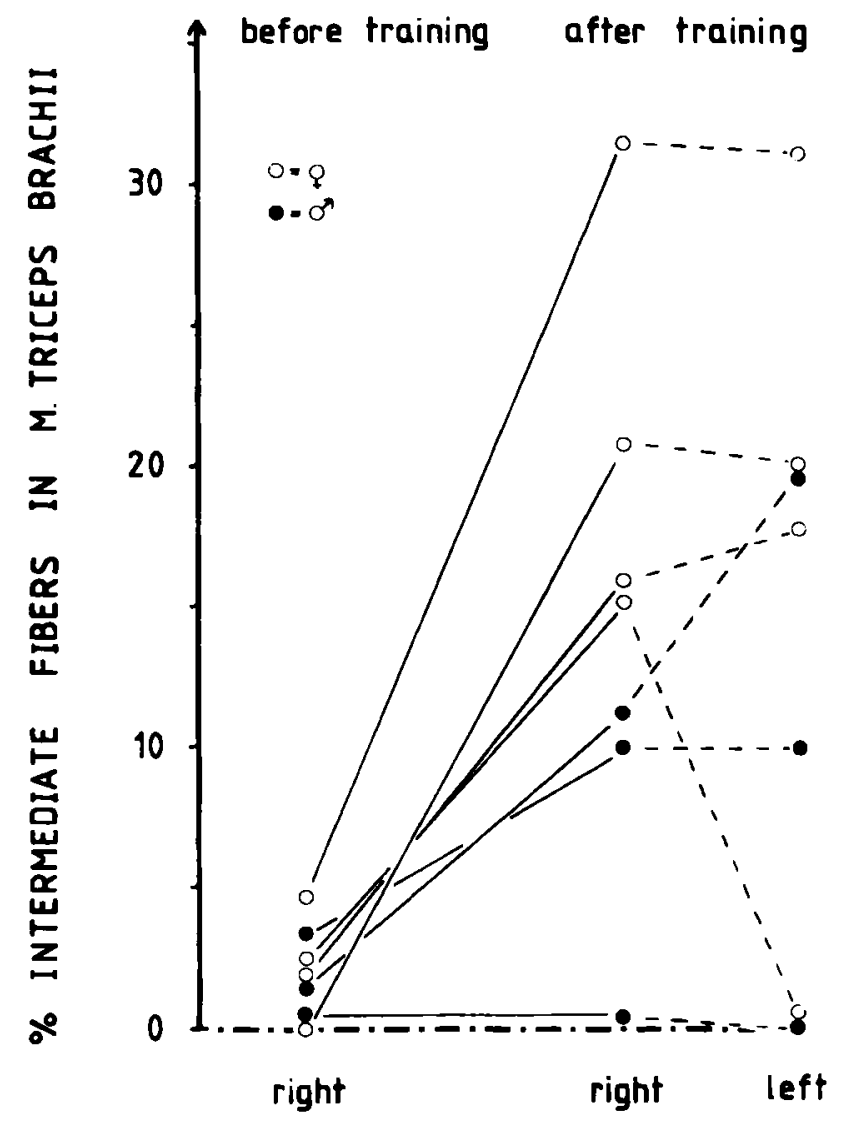

Figure 1. Individual percentages of intermediate fibers in the right and left $T B$ before and after training.

percentages of IM fibers are depicted in Fig. 1. The IM fibers were almost exclusively of the $2 \mathrm{C}$ type ${ }^{5,6}$ i.e., darkly stained for myofibrillar ATPase after alkaline preincubation, but intermediately stained after acid preincubation (Fig. 2). No morphological abnormalities were noted upon light microscopical inspection of the myofibrillar ATPase stainings, nor was there any obvious fiber type grouping evident.

\section{Discussion}

The present results provide two forms of evidence that the difference in fiber type distribution in the right TB before and after training is a result of the training program per se, and not related to nerve or fiber damage due to the biopsy sampling. The first form of evidence is that in five out of the six subjects in whom substantial increases of intermediate fibers are seen in the right TB, increases are also seen when comparing the left TB after training with the right TB at pretraining. The second form of evidence is that the posttraining fiber type distributions are similar in the right and left TB. A prerequisite for the preceding comparisons 

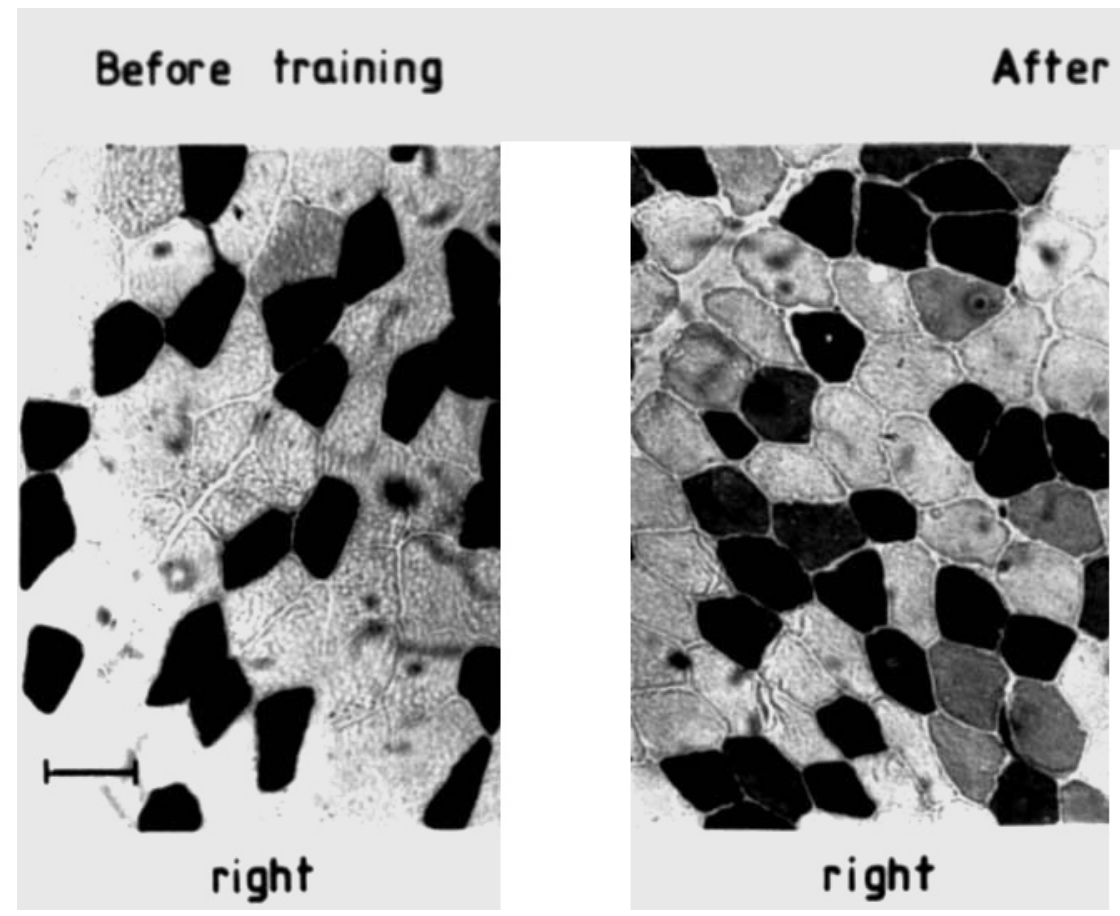

training

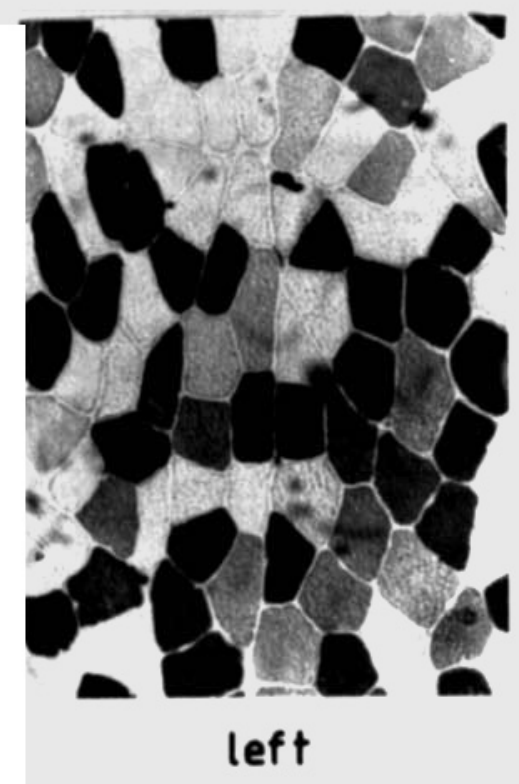

Higure 2. Staining for myotibrillar AlPase atter preincubation at pH 4.3. Ihe pictures were taken ot samples trom ib ot a temale subject in whom the percentage of intermediate fibers increased markedly during training. The intermdiate fibers were, with very few

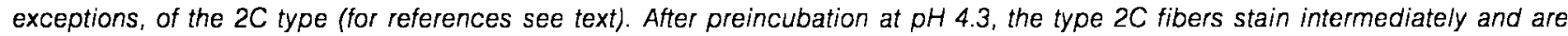
distinguishable from the other fiber types. (Type 1 fibers stain dark, type 2 fibers are unstained.) The length of the calibration bar corresponds to $0.1 \mathrm{~mm}$.

lies in the assumption that no difference existed between the right and left TB at pretraining. Although a difference in the distribution of type 1 and type 2 fibers has been noted between some dominant and nondominant hand and forearm muscles, ${ }^{9,11}$ a right-left symmetry has been shown in the upper arm and shoulder muscles, including $\mathrm{TB},{ }^{11}$ as well as in leg muscles. ${ }^{3}$ Furthermore, the authors have previously determined the fiber type distribution in the left TB of six untrained individuals. ${ }^{24}$ No intermediate fibers were observed. In the present study, the same frequency of type 1 fibers were seen in the right TB before and after training as in the left TB after training. Thus, the prerequisite for the present comparison seems to be fulfilled.

If the biopsy-taking procedure has influenced or caused the increase in intermediate fibers in the right $\mathrm{TB}$, a greater percentage of intermediate fibers would be expected in the right compared to the left TB after training. The risk that such a difference actually exists is not negligible. However, if the biopsy procedure has caused the increase in intermediate fibers, a decrease in both type 1 and type 2 fibers would be expected. As in the former study, which showed an increase in the intermediate fiber type after endurance training, ${ }^{2 \cdot 1}$ the increase in intermediate fibers was balanced by a decrease in type $2(\mathrm{~A}+\mathrm{B})$ fibers. Thus, with the preceding presented forms of evidence considered together, it seems hardly likely that the increased frequency of intermediate fibers is provoked or affected by the biopsy-taking. It is, therefore, concluded that prolonged endurance training may induce transformation of type 2 fibers into intermediate fibers.

\section{REFERENCES}

1. Bergström J: Muscle electrolytes in man. Scand J Clin Lab Invest (Suppl) 68:1-110, 1962.

2. Billeter R, Weber H, Lutz H, Howald H, Eppenberger HM, Jenny E: Myosin types in human skeletal fibres. Histochemistry 65:249-259, 1980.

3. Blomstrand E, Ekblom B: The needle biopsy technique for fibre type determination in human skeletal muscle-A methodological study. Acta Physiol Scand 116:437-442, 1982.

4. Brooke M, Kaiser K: Three "myosin ATPase" systems: The nature of their $\mathrm{pH}$ lability and sulfhydryl dependence. $J$ Histochem Cytochem 18:670-672, 1970.

5. Brooke M, Kaiser K: Muscle fiber types: How many and 
what kind. Arch Neurol 23:369-379, 1970.

6. Dubowitz V, Brooke M: Muscle Biopsy: A Modern Approach. London, W. B. Saunders, 1973, pp 50-51.

7. Eisen A, Karpati G, Carpenter S: Reserpine induced alteration of physiological properties and histochemical fibre types in rat skeletal muscle. Exp Neurol 46:554-565, 1975.

8. Engel W: The essentiality of histo- and cyto-chemical studies of skeletal muscle in the investigation of neuromuscular disease. Neurology (Minneap) 12:778-794, 1962.

9. Fugl-Meyer A, Eriksson A, Sjöström M, Söderström G: Is muscle structure influenced by genetical or functional factors? A study of three forearm muscles. Acta Physiol Scand 114:277-282, 1982.

10. Gomori G: The distribution of phosphatase in normal organs and tissues. J Cell Comp Physiol 17:71-83, 1941.

11. Josza L, Demel S, Reffy A: Fibre composition of human hand and arm muscles. Gegenbaurs Morphol Jahrb 127:3438,1981 .

12. Karpati $\mathbf{G}$, Engel $\mathrm{K}$ : Type grouping in skeletal muscles after experimental reinnervation. Neurology (Minneap) 18 $447-455,1968$

13. Kelly A, Rubinstein M: Patterns of myosin synthesis in regenerating normal and denervated muscles of the rat, in Pette D (ed): Plasticity of Muscle. Berlin, Walter de Gruyter \& Co, 1980, pp 161-175.

14. Kugelberg E: Histochemical composition, contraction speed and fatigability of rat soleus motor units. $J$ Neurol Sci 20:177-198, 1973 .

15. Kugelberg E: Adaptive transformation of rat soleus motor units during growth. Histochemistry and contraction speed. J Neurol Sci 27:269-289, 1976.
16. Lutz $H$, Weber E, Billeter R, Jenny E: Fast and slow myosin within single skeletal muscle fibers of adult rabbits. Nuture 281:142-144, 1979.

17. Mendenhall W: Introduction to Linear Models and the Design and Analysis of Experiments. Belmont, CA, Duxbury Press, 1968, pp 204-205.

18. Morris CJ: Human skeletal muscle fibre type grouping and collateral re-innervation. J Neurol Neurosurg Psychiatry 32: 440-444, 1969.

19. Morris CJ: The significance of intermediate fibres in reinnervated human skeletal muscle. $J$ Neurol Sci 11:123-136, 1970.

20. Müller W: Temporal progress of muscle adaptation to endurance training in hind limb muscles of young rats. A histochemical and morphometrical study. Cell Tissue Res 156:61-88, 1974

21. Padykula HA, Herman E: The specificity of the histochemical method for adenosine triphosphatase. $J$ Histochem $C y$ tochem 3:170-195, 1955.

22. Pette D, Müller W, Leisner E, Vrbová G: Time dependent effects on contractile properties, fibre population, myosin light chains and enzymes of energy metabolism in intermittently and continuously stimulated fast twitch muscles of the rabbit. Pfluegers Arch 364:103-112, 1976.

23. Riley D: Histochemical changes in ATPase activity during regeneration of adult skeletal muscle fibers. Exp Neurol 41 : $690-704,1973$.

24. Schantz $P$, Billeter $R$, Henriksson J, Jansson E: Training induced increase in myofibrillar ATPase intermediate fibers in human skeletal muscle. Muscle Nerve 5:628-636, 1982. 